\title{
Fingertip forces and completion time for index finger and thumb touchscreen gestures
}

Deanna S. Asakawa, George H. Crocker, Adam Schmaltz, and Devin L. Jindrich*

Department of Kinesiology, California State University San Marcos, 333. S. Twin Oaks Valley Rd., San Marcos, CA, USA

*Corresponding Author:

Devin L. Jindrich

Dept. of Kinesiology

California State University San Marcos

333 S. Twin Oaks Valley Rd.

San Marcos, CA 92096

$760750-7334$

djindrich@csusm.edu

keywords: hand; tapping; mobile computing; prehension; haptics

(C) 2016. This manuscript version is made available under the Elsevier user license http://www.elsevier.com/open-access/userlicense/1.0/ 


\section{Abstract}

2 Users actuate touchscreen computers by applying forces with their fingers to the

3 touchscreen, although the amount and direction of the force is unknown. Our aim was to

4 characterize the magnitude, direction and impulse of the force applied during single

5 finger (tapping and sliding in four directions) and two finger gestures (stretch and pinch).

6 Thirteen subjects performed repeated trials of each gesture. Mean $( \pm \mathrm{SD})$ resultant force

7 was $0.50(0.09) \mathrm{N}$ for tap, $0.79(0.32) \mathrm{N}$ to $1.18(0.47) \mathrm{N}$ for sliding gestures, $1.47(0.63) \mathrm{N}$

8 for pinch and 2.05(1.13) $\mathrm{N}$ for stretch. Mean resultant force was significantly less

$9 \quad(p<0.04)$ for tap than for all gestures except slide right. The direction of force application

10 was more vertical for the two-finger gestures as compared to the single- finger gestures.

11 Tap was the fastest gesture to complete at 133(83) ms, followed by slide right at 421(181)

12 ms. On average, participants took the longest to complete the stretch gesture at 920(398)

13 ms. Overall, there are differences in forces, force direction, and completion times among

14 touchscreen gestures that could be used to estimate musculoskeletal exposure and help

15 forge guidelines to reduce risk of musculoskeletal injury. 


\section{Introduction}

Human-computer interactions (HCI) such as keyboarding or mousing use repetitive motions and forces that increase risk for upper-extremity musculoskeletal disorders (Gerr et al., 2014, Gerr et al., 2006, Harris-Adamson et al., 2015). However, changes in both workplace and mobile computing technology are increasing the use of touchscreens in HCI (Nacher et al., 2015, Smith, 2015). Touchscreens are becoming increasingly common in both personal and workplace environments (Berolo et al., 2011, Duggan and Rainie, 2012, Smith, 2015). Therefore, determining whether touchscreens also involve exposure to motions and forces that may contribute to injury is important for evaluating the impacts of increased usage.

Several aspects of repetitive motion affect injury risk, including posture, movement extent, frequency, and force (Dennerlein, 2015, Kietrys et al., 2015). Touchscreen device design can change finger posture, affecting performance and potentially also injury risk for tapping tasks (Trudeau et al., 2016, Trudeau et al., 2013, Trudeau et al., 2012). Although tapping on a screen requires lower forces than on a keyboard, tapping on virtual keyboards also involves decreased performance and increased discomfort, suggesting that touchscreens may also present risks of musculoskeletal disorders (Kim et al., 2014).

Touchscreen interaction often involves more than tapping, and can include gestures such as swiping and pinching that result in large excursions of proximal finger joints (MCP; Asakawa et al., 2017). Moreover, the forces associated with non-tapping gestures are unknown. Because non-tapping gestures are less constrained than tapping, parameters such as gesture duration and force direction are also important. Therefore, determining force magnitude and direction, force impulse, and task 
completion time associated with non-tapping touchscreen gestures will be important for understanding the kinetics and potential injury risks of touchscreen use.

Our goal was to determine the parameters associated with force generation for seven common gestures on a touchscreen tablet computer. Based on preliminary work (Asakawa et al., 2014), published performance measures for touchscreen tapping (Kim and Song, 2014), as well as the kinematics and kinetics of the fingers and upper limb during tapping tasks (Asakawa et al., 2017, Dennerlein et al., 2007, Jindrich et al., 2004, Keenan and Massey, 2012), we developed hypotheses regarding the quantitative comparison of touchscreen tapping to other gestures. Specifically, we hypothesize that nontapping gestures will involve substantially smaller resultant forces, larger force impulses, and longer completion times than tapping on touchscreens. In total, we hypothesized that tapping involves the highest mean resultant force but the shortest completion time. We further hypothesize that the twofinger gestures will exert larger resultant forces per finger than single-finger sliding gestures. Because the biomechanics of the sliding motion requires a shear force be applied to the touchscreen, we expect shear forces in the plane of the touchscreen to be a greater percent of the resultant force for the sliding gestures as compared to the tap, pinch and stretch gestures. Finally, we predict that the direction of force application for single-finger sliding gestures would be along the slide direction. To test these hypotheses, we measured force and time parameters for tapping, sliding in four directions, pinching (zoom in) and stretching (zoom out) gestures.

\section{Methods}

\section{Subjects}

Thirteen unimpaired participants (7 female, 6 male; age 21-33 years) completed gestures on a 10.1-inch touchscreen tablet (Galaxy Tab 2, Samsung Group, Seoul, South Korea). All participants 
provided written informed consent for protocols approved by California State University San Marcos Institutional Review Board. No participant had a current upper limb injury or history of repetitive strain injury of the upper limb. All participants reported they had previous experience using touchscreen computing. Of the 13 participants, 11 were right-handed and 2 were left-handed. All participants completed gestures using their dominant hand. The subjects' average (SD) height was $1.68(0.10) \mathrm{m}$ and mass was 69.5 (14.4) kg. For all participants, we measured dominant hand length from the base of the palm to the tip of the third digit. Hand width was the distance from the second metacarpophalangeal joint (radial side) to the fifth metacarpophalangeal joint (ulnar side). Hand length and hand width averaged $17.8(1.0) \mathrm{cm}$ and $7.9(0.5) \mathrm{cm}$, respectively. Hand length percentiles for the participants ranged from a $3^{\text {rd }}$ percentile female to a $75^{\text {th }}$ percentile male (Gordon et al., 2012).

\section{Equipment and Setup}

Participants were seated in a rigid four-legged chair with seat height of $45.7 \mathrm{~cm}$ at a table with height $74.6 \mathrm{~cm}$. During data collection, the participants sat with their sternum aligned at the center of the tablet computer and both feet on the floor. All participants were able to complete gesture interactions comfortably without adjusting sitting posture. The tablet was placed $3.8 \mathrm{~cm}$ from the edge of the table with its long edge parallel to the subject's coronal plane (i.e., in landscape orientation, Fig. 1). Participants were not allowed to adjust the position of the touchscreen. The tablet had dimensions of $25.7 \times 17.5 \times 0.97 \mathrm{~cm}$, and weighed $0.58 \mathrm{~kg}$. We instructed subjects not to rest either hand or arm on the tablet or the table surface to prevent forces or moments not associated with gestures. We visually observed all participants during the data collection to ensure they did not touch the tablet computer except with the finger(s) needed for each gesture. The touchscreen was cleaned with a chamois cloth between participants or whenever necessary to keep the surface free of oils and maintain a consistent screen friction throughout data collection. Room temperature was controlled to maintain approximately 
$22^{\circ} \mathrm{C}\left(72^{\circ} \mathrm{F}\right)$ for all data collection.

A custom software application written for the Android operating system displayed $6 \times 6 \mathrm{~cm}$ square buttons in the center of the touchscreen for each gesture. The gestures included index finger tap, slide up, slide down, slide left, slide right, and index finger and thumb stretch (zoom an image in) and pinch (zoom an image out). The button size was selected to mimic common size slide or pinch gestures (e.g., for maps or book readers). Participants completed 11 consecutive repetitions of each of the 7 gestures presented in a randomized order. The four sliding gestures involved sliding a 1x6 cm bar across the $6 \times 6 \mathrm{~cm}$ box located in the center of the screen. The tap gesture involved tapping the tablet inside the 6x6 $\mathrm{cm}$ box. The pinch gesture involved placing the index finger and thumb at the upper right and lower left corner of the $6 \times 6 \mathrm{~cm}$ box and resizing the box by bringing the finger and thumb together. The stretch gesture required an opposite movement: starting with the finger and thumb together inside a smaller $1.5 \times 1.5 \mathrm{~cm}$ box and resizing to a $6 \times 6 \mathrm{~cm}$ box. Before each experiment, we provided a verbal description of the movements required for each gesture, and requested that participants complete gestures at a moderate, self-selected pace. Participants did not practice prior to data collection. No subject reported fatigue during the experiment.

\section{Data Collection}

The touchscreen tablet computer was mounted in a fitted rigid plastic case (Ballistic Case Co., Sunrise, FL) affixed to an aluminum plate $(24 \times 19 \times 0.4 \mathrm{~cm})$ bolted to a six degree-of-freedom load cell (JR3 Inc., Woodland, CA; Fig. 1). The load cell measured three-dimensional forces and torques applied to the tablet. When mounted on the load cell, the tablet touchscreen surface was $8.5 \mathrm{~cm}$ above the table top.

Calibration and validation indicated that measured forces were accurate to within $4 \%$ in the 
range associated with gestures. We calibrated the touchscreen force measurements by placing known vertical and horizontal weights at nine positions spanning the surface of the touchscreen. The force response of the transducer was linear for both force and moment. We computed a linear calibration matrix, and validated the matrix by placing known weights at positions on the screen not used for calibration. Force transducer data were recorded at $1000 \mathrm{~Hz}$ using Labview software (National Instruments Corporation, Austin, TX). During data collection, we used a manual trigger to identify the beginning and end of the gesture in the force recordings. We calculated forces from the transducer output using the calibration matrix, for each time sample between the beginning and end of each gesture using MATLAB (MathWorks, Natick, MA).

\section{Data Analysis}

We analyzed data using Microsoft Excel (Microsoft Corporation, Redmond, WA). Only gestures completed in one movement were analyzed. For example, we excluded gestures where the subject's finger(s) lifted off the tablet before completion. We did not analyze each subject's first trial for each gesture, resulting in analysis of a maximum of ten trials per subject per gesture.

We computed resultant force as the vector sum of the touchscreen forces in all three orthogonal directions. To filter out small force vibrations, we used only resultant forces above $0.20 \mathrm{~N}$ to compute average resultant force for each gesture. We computed shear force magnitude as the vector sum of forces in both planar directions. The normal force (perpendicular to the tablet's surface; Fz) was measured directly from the calibrated force signal. For two-fingered gestures, we calculated force per finger as the resultant force divided by two.

We calculated gesture completion time as the duration that a 9-ms moving average of the resultant force exceeded a $0.20 \mathrm{~N}$ threshold. We calculated resultant force impulse by integrating the 
resultant force-time curve using the trapezoid rule, for time points when the 9-ms moving average of the resultant force was greater than the $0.20 \mathrm{~N}$ threshold.

The average direction of force application was calculated as two separate angles: the shear force angle and the normal force angle. The shear force angle represented the direction of force in the plane of the touchscreen and was calculated from the mean forces in both planar directions (Fx and Fy, Fig. 2). A $0^{\circ}$ angle was parallel with the long axis of the tablet towards the subject's right $\left(180^{\circ}\right.$ to the subject's left). A $90^{\circ}$ angle was perpendicular to the long axis and directed away from the subject $\left(270^{\circ}\right.$ towards the subject; Fig. 2). For slide right, we used negative values for angles between $270^{\circ}$ and $360^{\circ}\left(-90^{\circ}\right.$ to $0^{\circ}$ ) for mean and SD calculations. The resultant force angle represented the vertical angle with respect to the screen. A $90^{\circ}$ angle was perpendicular and $0^{\circ}$ parallel to the touchscreen (Fig. 2). The mean vertical angle was calculated from the mean resultant and normal forces $(\mathrm{Fz})$.

\section{Statistical Analysis}

Peak force, average force, completion time and impulse were recorded for each participant performing each gesture. We calculated a mean, standard deviation, and coefficient of variation for each participant. We then computed a total mean for each measure across all 13 participants. Gestures were compared using repeated measures analysis of variance (RMANOVA) and Bonferonni's multiple pairwise comparison post-hoc tests. Correlation coefficients were computed for mean resultant forces and gesture completion times. Significance level was set at $\alpha=0.05$. All statistical analyses were completed using SPSS software version 22 (IBM Inc., New York, NY).

\section{Results}

\subsection{Gesture completion time was lowest for tapping}


Each gesture was, on average $( \pm \mathrm{SD})$, completed in less than $1 \mathrm{~s}$ with mean completion times ranging from 133(83) ms for tap to 920(398) ms for stretch (Fig. 3, Table 1). Consistent with our hypothesis, tapping had significantly faster completion time than all other gestures studied $(p<0.004)$. Slide right completion time of 421(181) ms, was significantly faster than pinch, stretch and slide down $(p<0.02)$. Completion times did not differ significantly among any other gestures.

\subsection{Mean resultant force was lowest for tapping}

Contrary to our hypothesis, mean resultant force for the tap gesture showed the lowest mean

force of all 7 gestures at $0.50(0.09) \mathrm{N}$ (Fig. 4A). Resultant force for tap was significantly less $(p<0.04)$ than resultant force for all gestures except slide right $(p=0.329)$. However, peak resultant force for tap was 1.44(0.73) $\mathrm{N}$ which was not significantly different from peak forces for other gestures, which ranged from $1.27(0.62) \mathrm{N}$ for slide right to $3.2(1.6) \mathrm{N}$ for stretch.

\subsection{Mean per-finger resultant force for pinch and stretch did not differ from forces in other gestures}

The two-finger gestures of pinch and stretch did not involve more average resultant force per finger than single-finger gestures ( $\mathrm{p}>0.05$ for comparisons with all other gestures). Mean total resultant force exerted on the touchscreen was highest for stretch (total force for both fingers of 2.05(1.13 N)). However, resultant force per finger for stretch was only $1.02(0.57) \mathrm{N}$ and for pinch was $0.74(0.31) \mathrm{N}$, not significantly different from any other gesture. Slide up measured the highest mean resultant force of the four sliding directions at $1.18(0.47) \mathrm{N}$ (Fig. 4A). Slide right showed the lowest peak resultant force with $0.79(0.38) \mathrm{N}$ of the sliding directions. Slide right was performed with significantly less peak resultant force than slide up, pinch and stretch $(p<0.03)$. Slide up had the highest mean force per finger when comparing force for the index finger only gestures to mean force per finger for two-finger gestures. Therefore, the data do not support our hypothesis that two-finger gestures exerted more 
average force per finger than single-finger gestures.

\subsection{Sliding gestures had a greater percentage of their total force as shear force}

For all sliding gestures, the component of shear force was greater than the normal force component (Fig. 4B). Conversely, for pinch, stretch and tap, the normal force was greater than the shear force. Shear forces were largest for the sliding gestures, ranging from $0.62(0.34) \mathrm{N}$ for slide right to 0.90(0.38) $\mathrm{N}$ for slide up, as compared to other gestures (Fig. 5A). Shear force for tapping, 0.13(0.06) N, and pinching, $0.28(0.20) \mathrm{N}$ were significantly smaller $(p<0.04)$ than the four sliding gestures but were not different from the shear force for stretch, $0.37(0.21) \mathrm{N}$. Shear forces in the plane of the screen were $20 \%$ and $19 \%$ of the normal force for pinch and stretch, respectively, resulting in vertical angles for pinch and stretch of $78.7^{\circ}$ to $79.3^{\circ}$ (Fig. 4B). For the sliding gestures, shear forces averaged $130 \%$ to $158 \%$ of the normal force, resulting in a vertical angle between $32.4^{\circ}$ and $37.5^{\circ}$. For tapping, the shear force was $42 \%$ of the normal force resulting in mean normal force angle of $67.0^{\circ}$.

The direction of the shear force application was coincident with the direction of slide for all four sliding gestures (Fig. 5B). The mean direction of shear force application for all subjects was within $1^{\circ}$ of the direction of the slide for the left and right sliding gestures. Slide up and slide down had a mean shear force direction of $92.4(3.8)^{\circ}$ and $276.2(3.1)^{\circ}$, respectively, which corresponds with $2.4(3.8)^{\circ}$ to the left of up and $6.2(3.1)^{\circ}$ to the right of down.

Tapping and both two-finger gestures had higher variability for shear force directions compared to sliding gestures. Standard deviations ranged from $21.2^{\circ}$ for stretch to $41.2^{\circ}$ for tap. In contrast, the sliding gestures demonstrated standard deviations between $2.8^{\circ}$ and $5.1^{\circ}$. Across participants, the sliding gestures showed the least variability in the way forces were applied to the touchscreen. 


\subsection{Force impulse differed among gestures}

Each gesture had a characteristic force versus time profile (Fig. 6). Impulse measurements showed that tap had the least impulse of $0.071(0.054)$ Ns (Fig .7). Tap had significantly smaller $(p<0.03)$ resultant force impulse than pinch, slide down and slide up (Fig. 7). The stretch gesture had a resultant force impulse of 2.250(2.111) Ns, but was not significantly different than any other gesture. Therefore, the data did not support the hypothesis that non-tapping gestures produce smaller force impulse than tapping.

\subsection{Variability for resultant force was lowest for tapping}

Tapping had the lowest coefficient of variation (18\%) for mean resultant force of all gestures, which ranged from $40 \%$ for slide up to $55 \%$ for stretch (Table 1). Taken together, tap had the lowest and least variable mean resultant force among all gestures studied. In contrast, tap had the highest coefficient of variation for completion time (63\%). Pinch was the least variable gesture for completion time with a coefficient of variation of $36 \%$. The other gestures ranged from $39 \%$ variability in completion time for slide down to $53 \%$ for slide left.

\subsection{Correlation between gesture time and force}

Mean gesture force was correlated with gesture completion time $(\mathrm{r}=0.9477 ; p=0.001)$. Gestures with longer durations had higher mean forces (Fig. 8). When using mean force per finger for pinch and stretch, the correlation of force and completion time was not significant, $(\mathrm{r}=0.641, p=0.121)$.

\section{Discussion}

Measuring fingertip forces, force directions, completion times and force impulses during 
touchscreen gesturing led to several important findings. Both the amount and duration of force were different among gestures, potentially influencing musculoskeletal exposure to forces. Although stretch had the highest mean resultant force and longest completion time of the gestures studied, stretch did not have the highest force per finger. Tapping demonstrated the shortest completion time among all gestures, and tapping also did not involve high forces. Mean resultant force for tapping was lower than all other gestures except slide right. Slide up had the highest resultant force per finger. Although twofinger gestures exerted higher total force on the touchscreen, if forces were distributed equally between fingers, total loading per finger was approximately equal across all gestures. However, force direction was not consistent across conditions: shear forces were greater during sliding gestures.

Some limitations should be considered when evaluating the results of our study. First, we recorded a single resultant force on the touchscreen for the two-finger gestures, and could not separate forces from the thumb and index finger for the pinch and stretch gestures. We assumed that load was distributed evenly between both fingers, and divided the resultant force by two to compute average force per finger. However, fingers may not evenly distribute load, nor necessarily work together to complete a gesture. Uneven distribution could reduce the forces generated by one finger, and opposition could increase forces on both fingers without affecting the resultant force. Therefore, finger forces could be lower than half of the resultant forces for one finger, or higher for one or both fingers. Our conclusions are therefore conservative: forces for both fingers are unlikely to be lower than half the resultant force, although they could be higher.

Second, for this study the participants completed the gestures at a self-selected moderate pace. We did not give verbal instructions to complete gestures as quickly as possible. Therefore, the completion times we measured may be longer than gestures intended to be rapid (Kim et al., 2014, Asakawa et al., 2017). However, a self-selected moderate pace may be representative of common 
interactions with touchscreens.

Tapping on a touchscreen was rapid relative to other gestures. However, the touchscreen tapping time that we measured was substantially longer than durations measured when tapping on virtual keyboards ( $<50 \mathrm{~ms}$; Kim et al., 2014). Our results are consistent with those for tapping on keyswitches, where tapping on an isolated keyswitch ( $225 \mathrm{~m}$; Jindrich et al., 2004) involves substantially longer duration than keystrokes during typing ( 100 ms for keyboarding; Rempel et al., 1994). The discreet movements of a single tap may be different from the more continuous movements of typing for both physical and virtual keyboards.

Correlation between force and completion time was significant, potentially due to tap having low force and completion time and stretch having the highest total force and completion time of all gestures studied. Future work measuring different types of slides may be necessary to determine whether a general relationship exists between force and completion time among sliding gestures. A correlation between force and completion time could have an important practical application. Completion time is easily measured and could be used to estimate force, and in turn exposure, during touchscreen gesturing.

Our data do not support the hypothesis that tapping involves larger resultant forces than swiping and two-fingered gestures. Upper limb and finger kinematics could play a role in force generation (Dennerlein et al., 2007, Edsfeldt et al., 2015). We hypothesized that tapping involved high forces due to the potential for vertical wrist motion, as opposed to the distal finger joint motion used for pinch and stretch (Asakawa et al., 2017). However, the forces measured for tapping in this study were lower than sliding and stretching gestures, suggesting that future work is needed to quantify upper limb kinematics during gesturing to more completely understand the biomechanics of force generation on the touchscreen. 
Typing forces for virtual keyboards are significantly lower than for traditional keyboards (Kim et al, 2014). The forces associated with activating touchscreen buttons were lower even than those associated with keystrokes on virtual keyboards ( $0.5 \mathrm{~N}$ vs. $\sim 0.75 \mathrm{~N}$; Kim et al., 2014). Although activating touchscreens does not depend on force, forces associated with tapping reflect several aspects of the movement. For example, keyboarding may involve more muscle activation or co-activation than tapping, because other fingers must be prepared to tap if necessary. Typing typically involves both hands and therefore a potentially different posture of the fingers, wrist and upper limb. Moreover, the cost of failure may be less for tapping than for keyboarding, where a missed stroke can result in the need to correct an entire word.

Similar to keyboarding, subjects were not required to hold or support the tablet. Kietrys et al. (2015) found that as touchscreen size increased, people were more likely to set a mobile computing device on a supporting surface. However, small mobile computing devices are often held by the hand contralateral to the tapping hand, and device support may affect the magnitude of forces applied (Gold et al., 2012, Jonsson et al., 2011, Kietrys et al., 2015, Trudeau et al., 2016). Tapping with different fingers may also involve different forces. Single finger tapping on touchscreens may involve different patterns of movement, coordination, and control than keyboard typing, potentially explaining why single-finger touchscreen tapping involves lower forces than virtual keyboard typing (Kim et al, 2014).

Musculoskeletal injuries including tendonitis and arthritis of the hand have been linked to repetitive use of the finger and thumb joints (Gerr et al., 2006, Brewer and Storey, 2016). Therefore, it is critical to understand the magnitude and direction of forces experienced by the digits of the hand during touchscreen use (Dennerlein, 2015). Shear forces experienced with sliding gestures could contribute to increased abduction/adduction loads on the fingers, while normal forces during pinch and stretch could contribute to flexion/extension loads on the fingers that are greater than loads during tapping. Injury to 
the joints of the hand could lead to difficulty with activities of daily living. For example, Gehrmann et al. (2010) showed arthritis of the thumb carpometacarpal joint of can loss of motion in both extension and adduction. Knowledge of the forces applied during touchscreen gestures can contribute to creating ergonomic guidelines aimed to reduce risk of injury to the joints and soft tissues of the fingers and hand.

Tapping was the least variable gesture for force measurements, but the most variable gesture for completion time measurements. Stretch was most variable gesture for force. Different gestures demonstrated different variability for force characteristics and completion times. Future work will be necessary to explore the variability among gestures, as high variability in segment interaction has been linked to less risk of overuse injury (Hamill et al., 2012).

The force measurements presented by this study are the first quantitative measurements of touchscreen gestures, and contribute to understanding gesturing motions that are a common part of daily technology interactions for much of the population (Berolo et al., 2011, Nacher et al., 2015, Smith, 2015).

Some practical implications of our results can be drawn from the comparison of kinetic measures among gestures. For example, when comparing the four orthogonal directions of sliding or swiping, the lowest mean resultant force was measured for slide right, the highest force for slide up. Slide right was significantly less force than slide up, pinch and stretch. This may indicate that slide right would be the preferred direction of sliding if the goal of software is to minimize the amount of force applied repetitively by the fingers. In addition, this work demonstrated that the force-time profiles were characteristic for each gesture. Force magnitude, direction and impulse can be used to estimate comparative musculoskeletal exposure among gestures during software application design.

Overall, measurement of touchscreen gesture forces and time parameters led to several findings 
with importance to characterizing and evaluating differences among finger kinetics commonly used for HCI. These data and findings provide a first step toward understanding the biomechanical differences among different touchscreen gestures, and the potential musculoskeletal risks associated with use of repetitive interactions with a touchscreen for tapping, sliding, pinch and stretch gestures.

\section{Acknowledgements}

This work is funded by the National Science Foundation (0964220). We thank Jack Dennerlein and Jong Hwa Lee for their contributions to this project. 


\section{References:}

Asakawa DS, Dennerlein JT, Jindrich DL. Index finger and thumb kinematics and performance measurements for common touchscreen gestures. Applied Ergonomics. 2017;58:176-81.

Asakawa DS, Dennerlein JT, Jindrich DL. Comparison of Task Completion Time, Finger Joint Angles, and the Pressure Applied by the Fingers for 7 Common Gestrues on a Touchscreen Computing Device. $7^{\text {th }}$ World Congress of Biomechanics, Boston, MA. 2014.

Berolo S, Wells RP, Amick BC, 3rd. Musculoskeletal symptoms among mobile hand-held device users and their relationship to device use: A preliminary study in a Canadian university population. Appl Ergon. 2011;42:371-8.

Brewer PE, Storey PA. The hand and wrist in rheumatoid and osteoarthritis. Surgery. 2016;34(3):144151

Dennerlein JT. The state of ergonomics for mobile computing technology. Work. 2015;52:269-77.

Dennerlein JT, Kingma I, Visser B, van Dieen JH. The contribution of the wrist, elbow and shoulder joints to single-finger tapping. Journal of Biomechanics. 2007;40:3013-3022

Duggan M, Rainie L. Cell Phone Activities Pew Research Center's Internet \& American Life Project2012. p. 20.

Edsfeldt S, Rempel D, Kursa K, Diao E, Lattanza L,. In vivo flexor tendon forces generated during different rehabilitation exercises. J Hand Surg Eur Vol. 2015; 40(7):705-10

Gehrmann SV, Tang J, Li ZM, Goitz RJ, Windolf J, Kaufmann RA. Motion Deficit in the thumb in CMC joint arthritis. J Hang Surgery. 2010;35A:1449-1453.

Gerr F, Fethke NB, Merlino L, Anton D, Rosecrance J, Jones MP. A prospective study of musculoskeletal outcomes among manufacturing workers: I. Effects of physical risk factors. Human factors. 2014;56:112-30.

Gerr F, Monteilh CP, Marcus M. Keyboard use and musculoskeletal outcomes among computer users. Journal of Occupational Rehabilitation. 2006;16:259-71.

Gold JE, Driban JB, Thomas N, Chakravarty T, Channell V, Komaroff E. Postures, typing strategies, and gender differences in mobile device usage: an observational study. Applied Ergonomics. 2012;43:408-412.

Gordon CC, Blackwell CL, Bradtmiller B, Parham JL, Barrientos P, Paquette SP, Corner BD, Carson JM, Venezie JC, Rockwell BM, Mucher M, Kristensen S, 2012 Anthropometric survey of U.S. army personnel:methods and summary statistics. Technical Report US. Army Natick Soldier Research, Development and Engineering Center, Natick, MA. 2012.

Hamill J, Palmer C, and Van Emmerik REA, Coordinate variability and overuse injury. Sports Medicine, Arthoscopy, Rehabilitation, Therapy \& Technology. 2012; 4:45

Harris-Adamson C, Eisen EA, Kapellusch J, Garg A, Hegmann KT, Thiese MS. Biomechanical risk factors for carpal tunnel syndrome: a pooled study of 2474 workers. Occupational and environmental medicine. 2015;72:33-41.

Jindrich $D L$, Balakrishnan AD, Dennerlein JT. Finger joint impedance during tapping on a computer keyswitch. J of Biomechanics. 2004; 37:1589-1596

Jonsson P, Johnson PW, Hagberg M, Forsman M. Thumb joint movment and muscular activity during mobile phone texting - a methodological study. J of Electromyography and Kinesiology. 2011; 363370. 
Keenan KG, Massey WV. Control of fingertip forces in young and older adults pressing against fixed low- and high- friction surfaces. PloSOne. 2012; 7 (10):e48193.

Kietrys DM, Gerg MJ, Dropkin J, Gold JE. Mobile input device type, texting style and screen size influence upper extremity and trapezius muscle activity, and cervical posture while texting. Applied Ergonomics. 2015;50:98-104.

Kim $\mathrm{H}$, Song $\mathrm{H}$. Evaluation of the safety and usability of touch gestures in operating in-vehicle information systems with visual occlusion. Applied Ergonomics. 2014; 45:789-798.

Kim JH, Aulck L, Bartha MC, Harper CA, Johnson PW. Differences in typing forces, muscle activity, comfort, and typing performance among virtual, notebook, and desktop keyboards. Appl Ergon. 2014;45:1406-13.

Nacher V, Jaen J, Navarro E, Catala A, González P. Multi-touch gestures for pre-kindergarten children. International Journal of Human-Computer Studies. 2015;73:37-51.

Rempel, D., Dennerlein, J., Mote Jr., C.D., Armstrong, T. A method of measuring fingertip loading during keyboard use. Journal of Biomechanics. 1994;27 (8), 1101-1104

Smith A. U.S. smartphone use in 2015. Pew Research Center; 2015.

Trudeau MB, Asakawa DS, Jindrich DL, Dennerlein JT. Two-handed grip on a mobile phone affords greater thumb motor performance, decreased variability, and a more extended thumb posture than a one-handed grip. Applied Ergonomics. 2016;52:24-8.

Trudeau MB, Catalano PJ, Jindrich DL, Dennerlein JT. Tablet Keyboard Configuration Affects Performance, Discomfort and Task Difficulty for Thumb Typing in a Two-Handed Grip. PLoS One. 2013;8:e67525.

Trudeau MB, Young JG, Jindrich DL, Dennerlein JT. Thumb motor performance varies with thumb and wrist posture during single-handed mobile phone use. J Biomech. 2012;45:2349-54. 


\section{Captions}

Fig. 1. Experimental set-up. Participants were seated with the touchscreen tablet centered on a table in front of them. The tablet was affixed rigidly to a force transducer that measured the forces applied on the touchscreen.

Fig. 2. A. Top view of touchscreen tablet computer showing shear force angle convention. B. Side view of touchscreen tablet computer (1) and load cell (2) showing a resultant force $\left(\mathrm{F}_{\mathrm{r}}\right)$ being applied to the screen with the shear force $\left(\mathrm{F}_{\text {shear }}\right)$ and normal force $\left(\mathrm{F}_{\text {normal }}\right)$ components. The angle between the resultant force and the touchscreen was defined as the vertical angle.

Fig. 3. Mean completion time for one-finger tapping (black bar), two-finger sliding (white bars), and one-finger sliding (gray bars) gestures. Values are means $+\mathrm{SD}$ for 13 subjects. $R$ and $T$ denote significant differences from slide right and tap, respectively.

Fig. 4. A. Mean resultant force for one-finger tapping (black bar), two-finger sliding (white bars), and one-finger sliding (gray bars) gestures. Values are means $+\mathrm{SD}$ for 13 subjects. $R$ and $T$ denote significant differences from slide right and tap, respectively. B. Mean magnitude with respect to the touchscreen of the resultant force plotted as a function of shear force on the horizontal axis and normal force on the vertical axis.

Fig. 5. A. Mean shear force for one-finger tapping (black bar), two-finger sliding (white bars), and onefinger sliding (gray bars) gestures. Values are means $+\mathrm{SD}$ for 13 subjects. $P, T$ and $U$ denote significant 
differences from pinch, tap, and slide up, respectively. B. Mean magnitude and direction of the shear force with respect to the touchscreen for the sliding (gray circles) and tapping (black triangle) gestures.

Fig. 6. Representative force-time profiles for each touchscreen gesture from one participant.

Fig. 7. Mean resultant impulse for two-finger (white bars), one-finger sliding (gray bars) and one-finger tapping (black bar) gestures. Values are means + SD for 13 subjects. $P$ and $T$ denote significant differences from pinch and tap, respectively.

Fig. 8. Completion time versus mean resultant force for one-finger tapping (black triangle), two-finger sliding (white squares), and one-finger sliding (gray circles). 
Fig. 1.

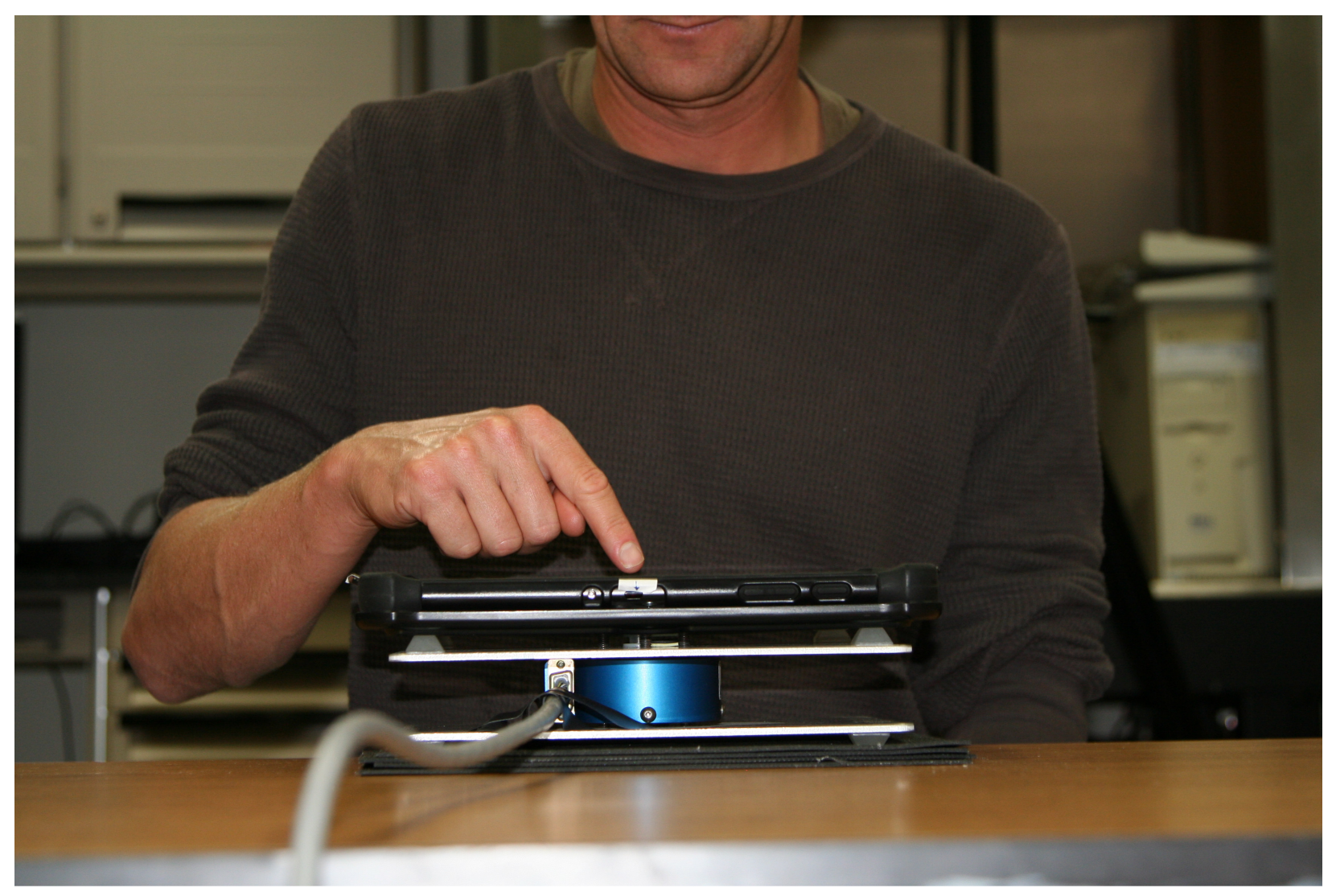


Fig. 2.

A

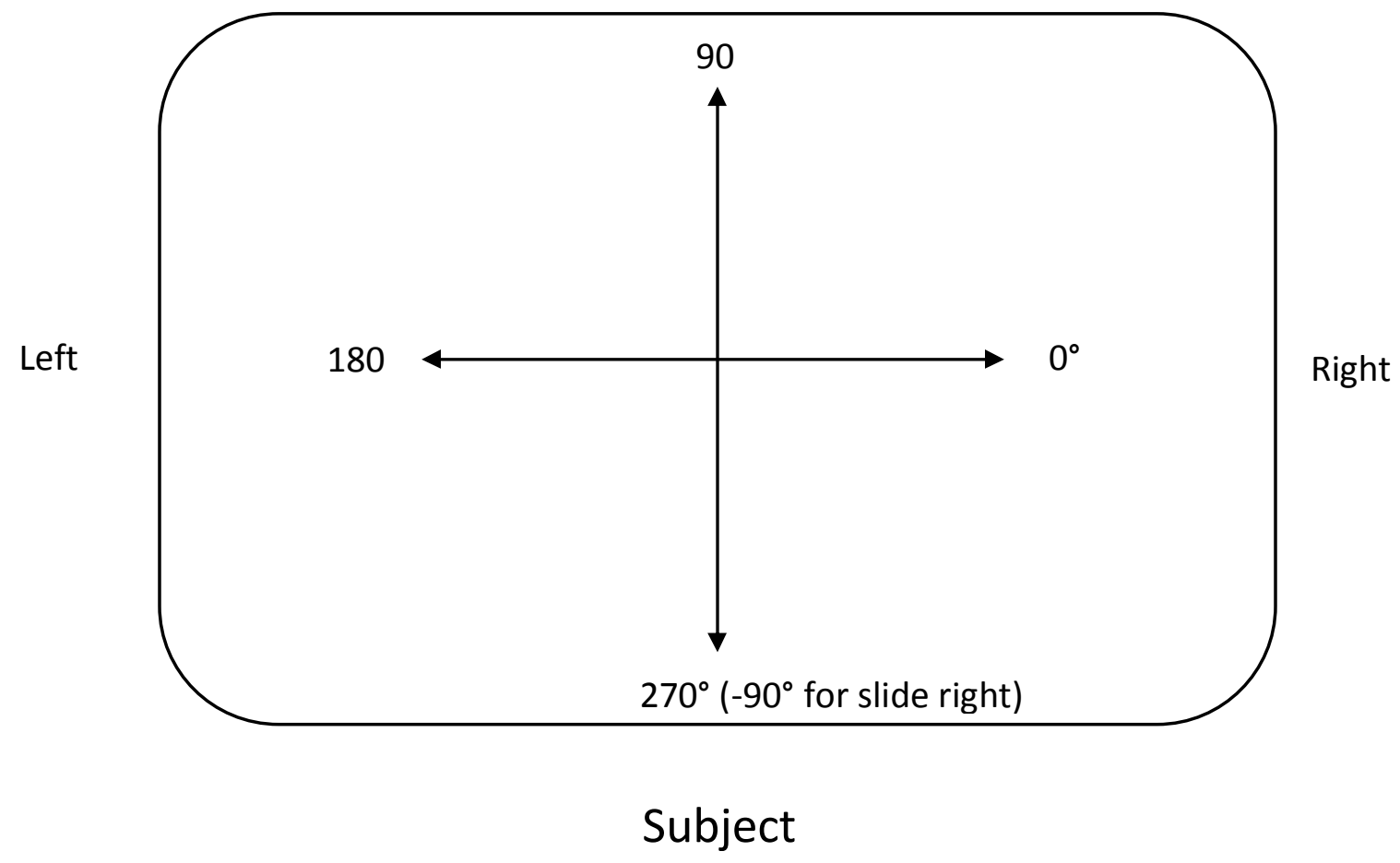

B

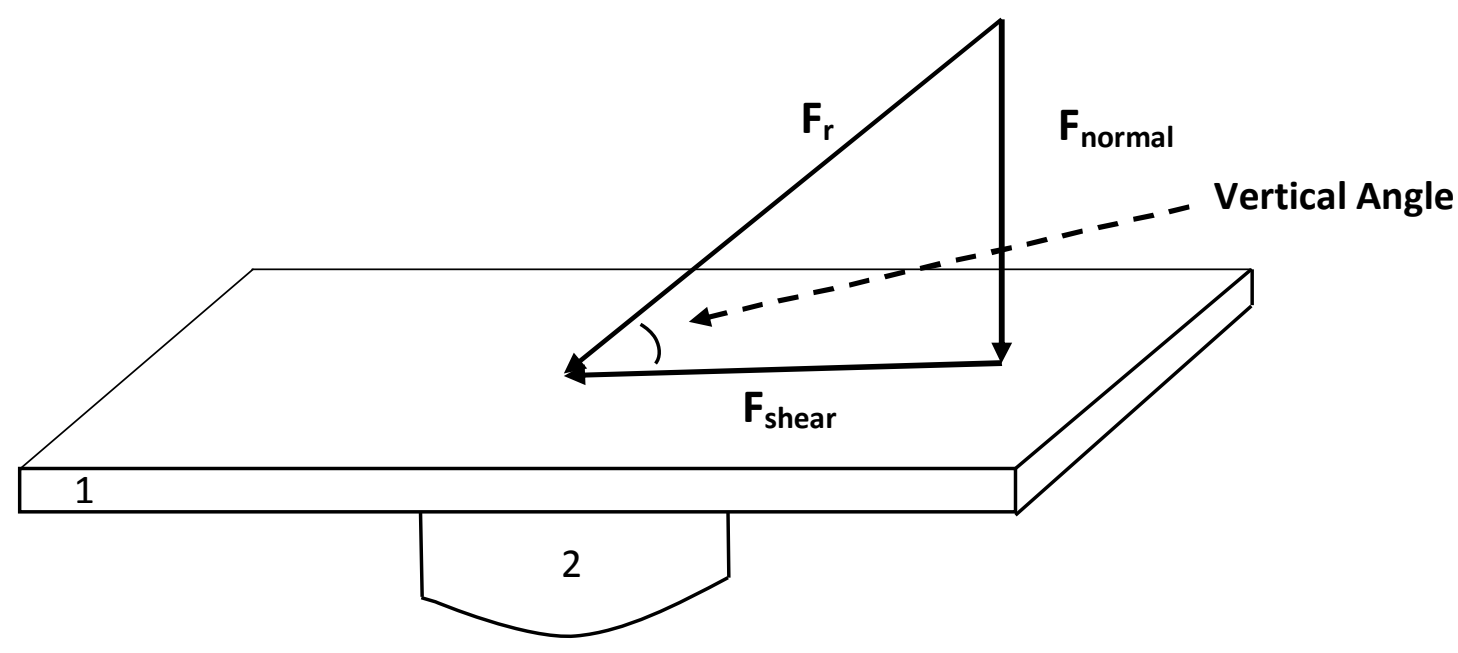


Fig. 3.

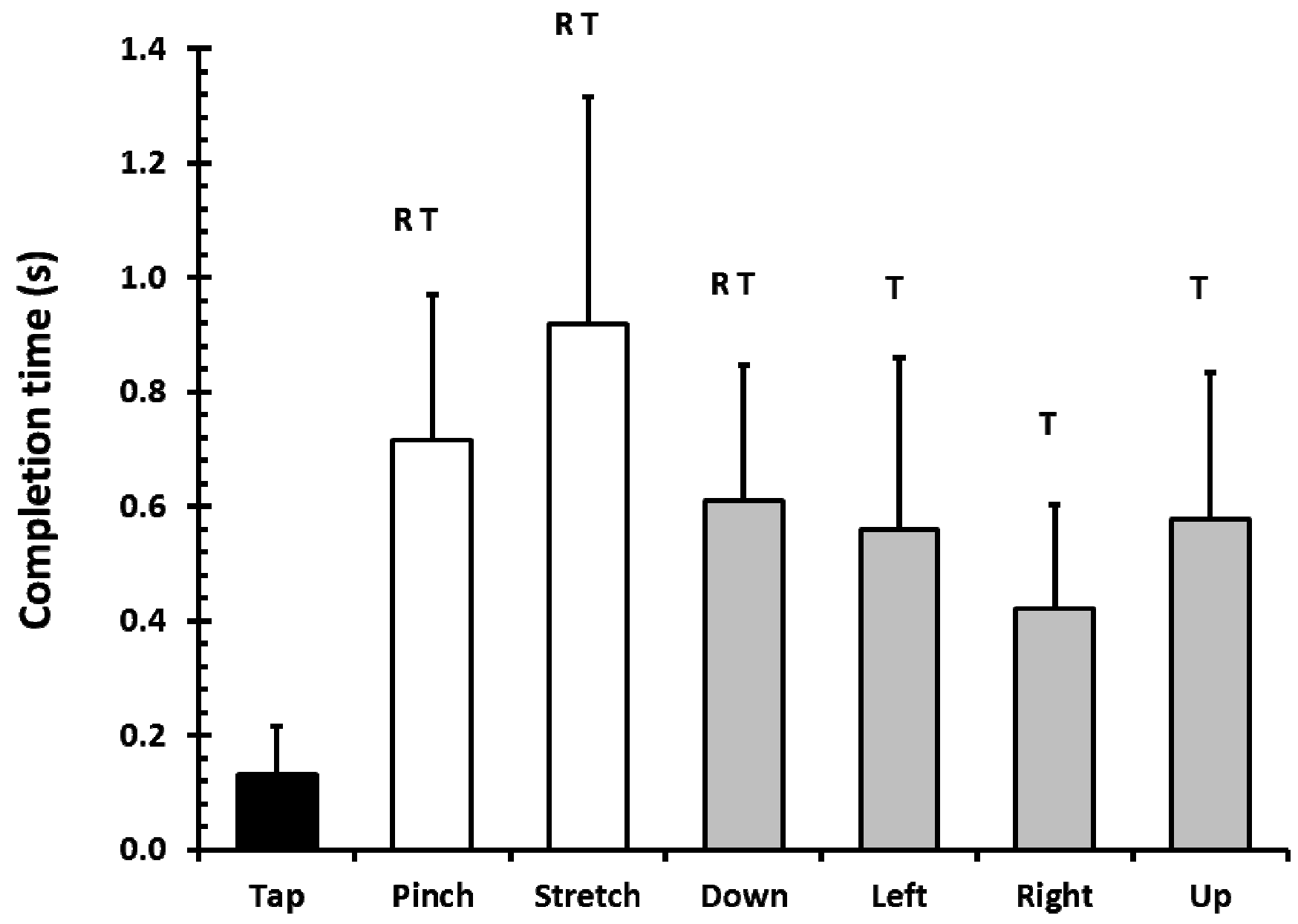


Figure 4.
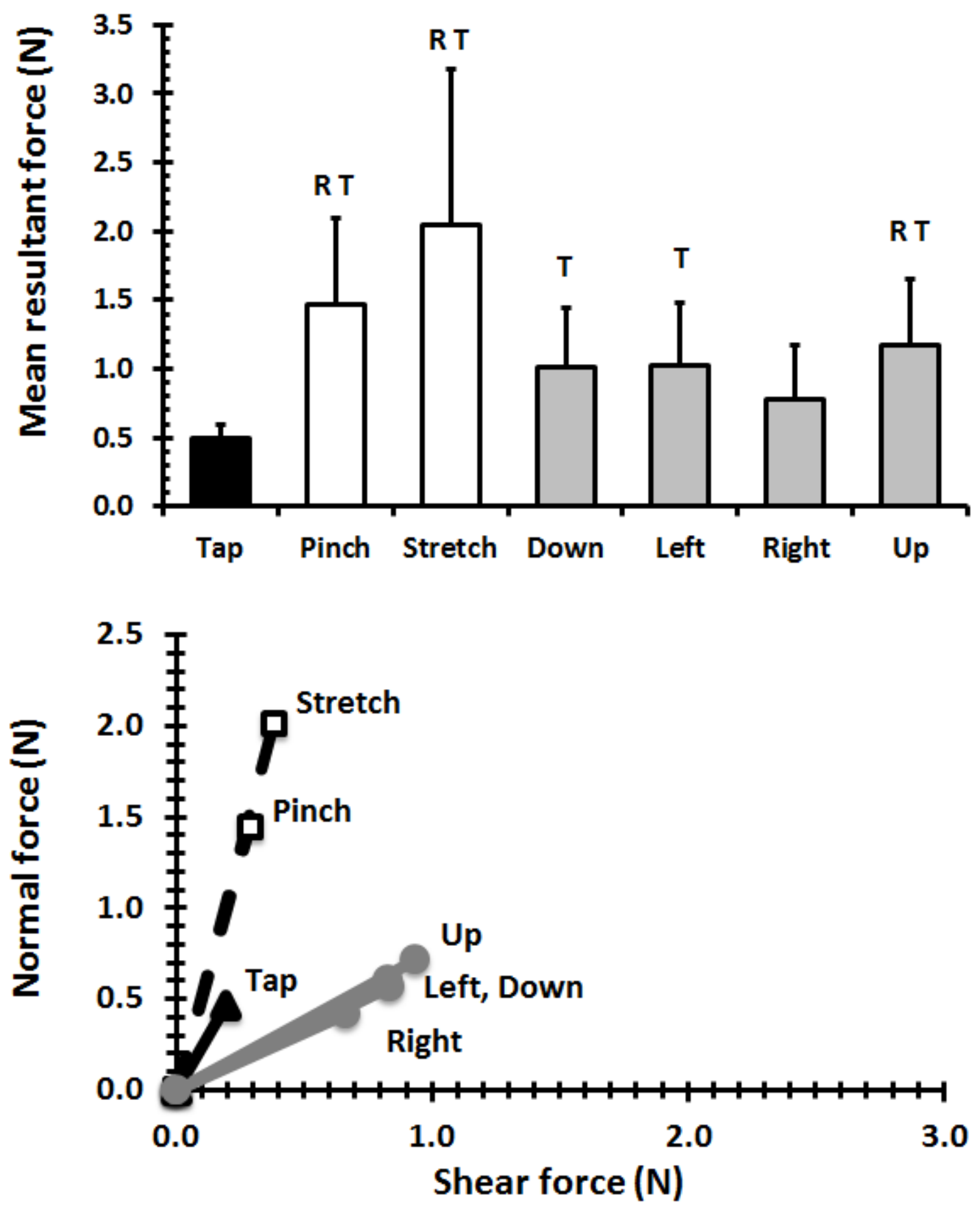
Fig. 5.

A
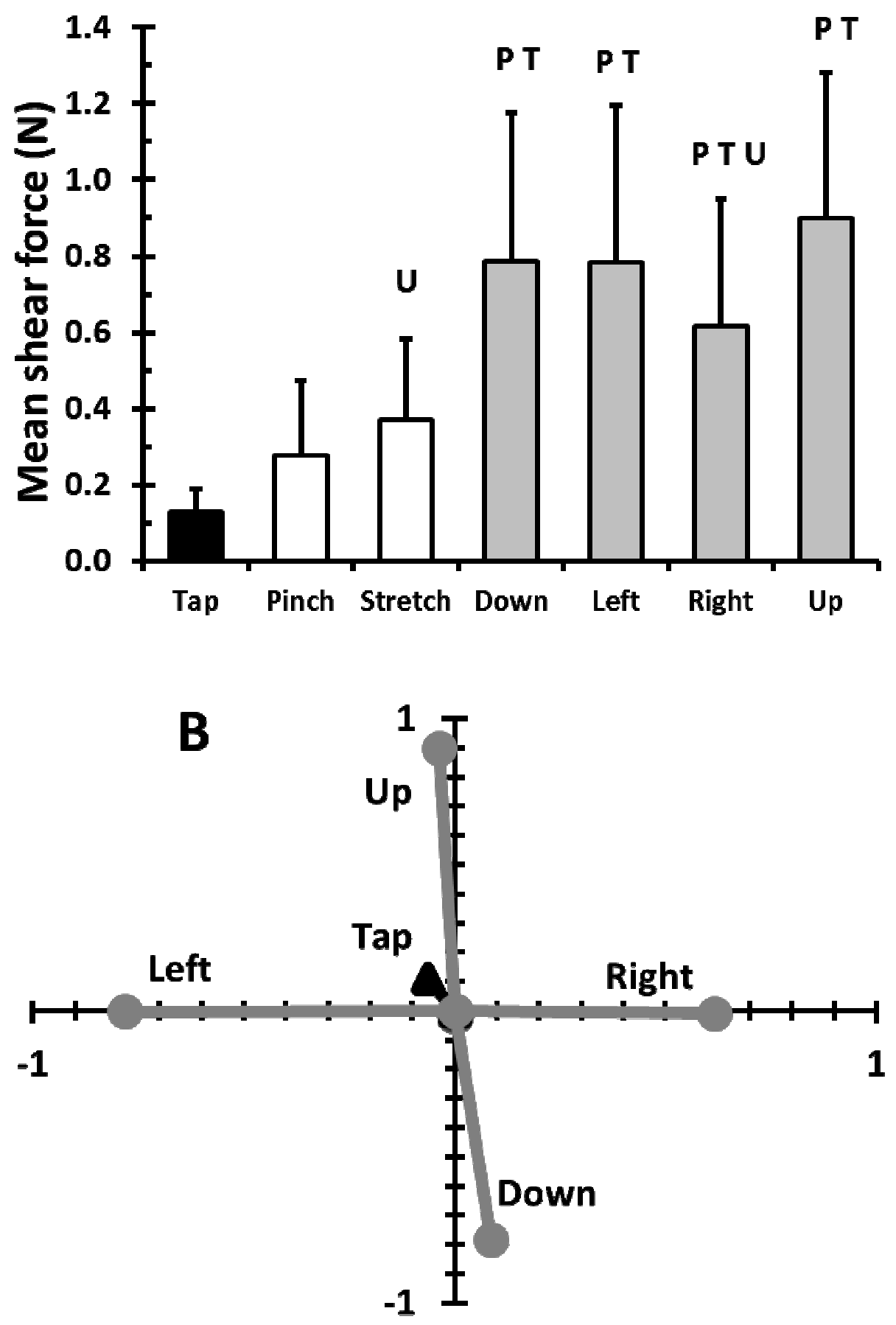
Fig. 6.
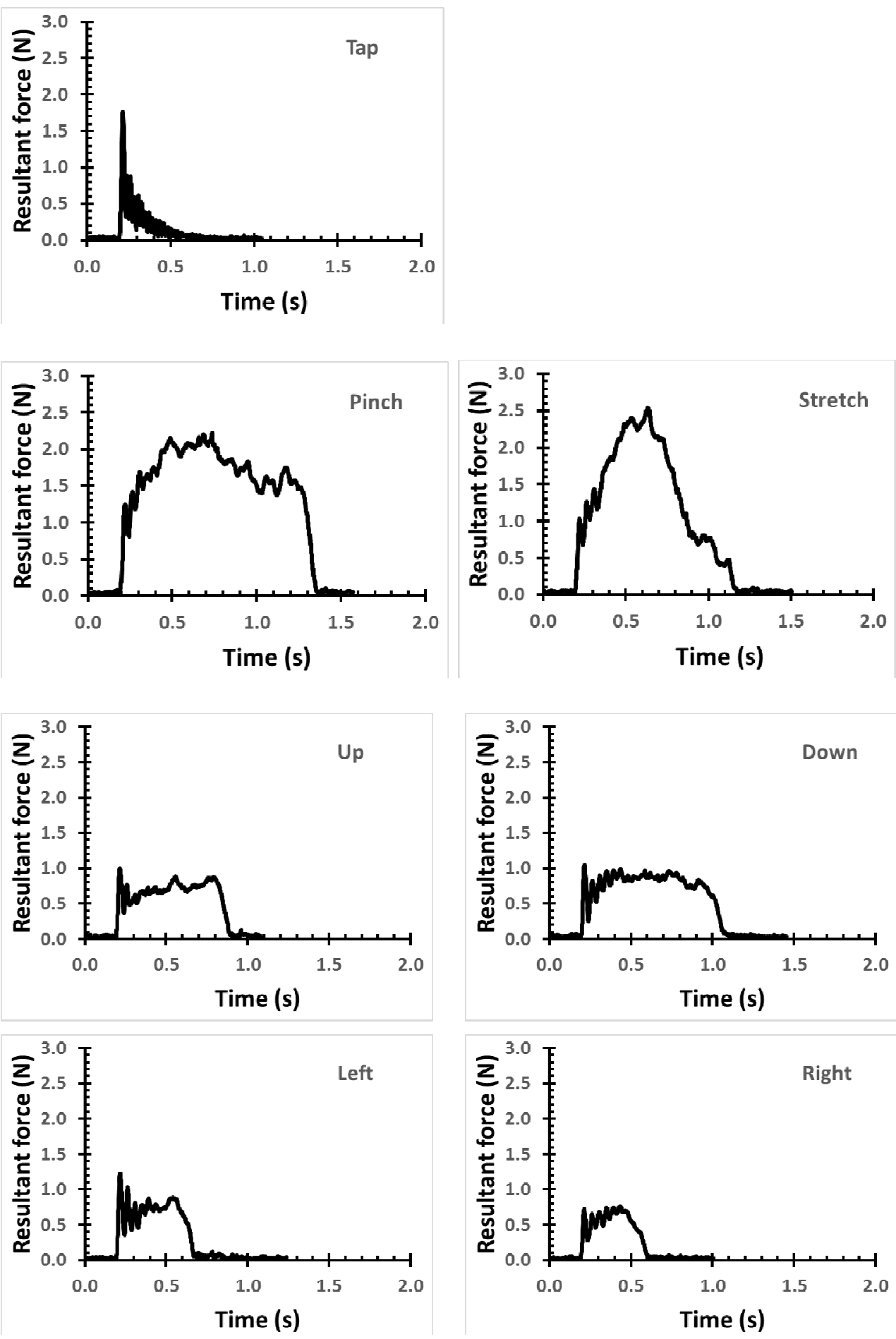
Fig. 7.

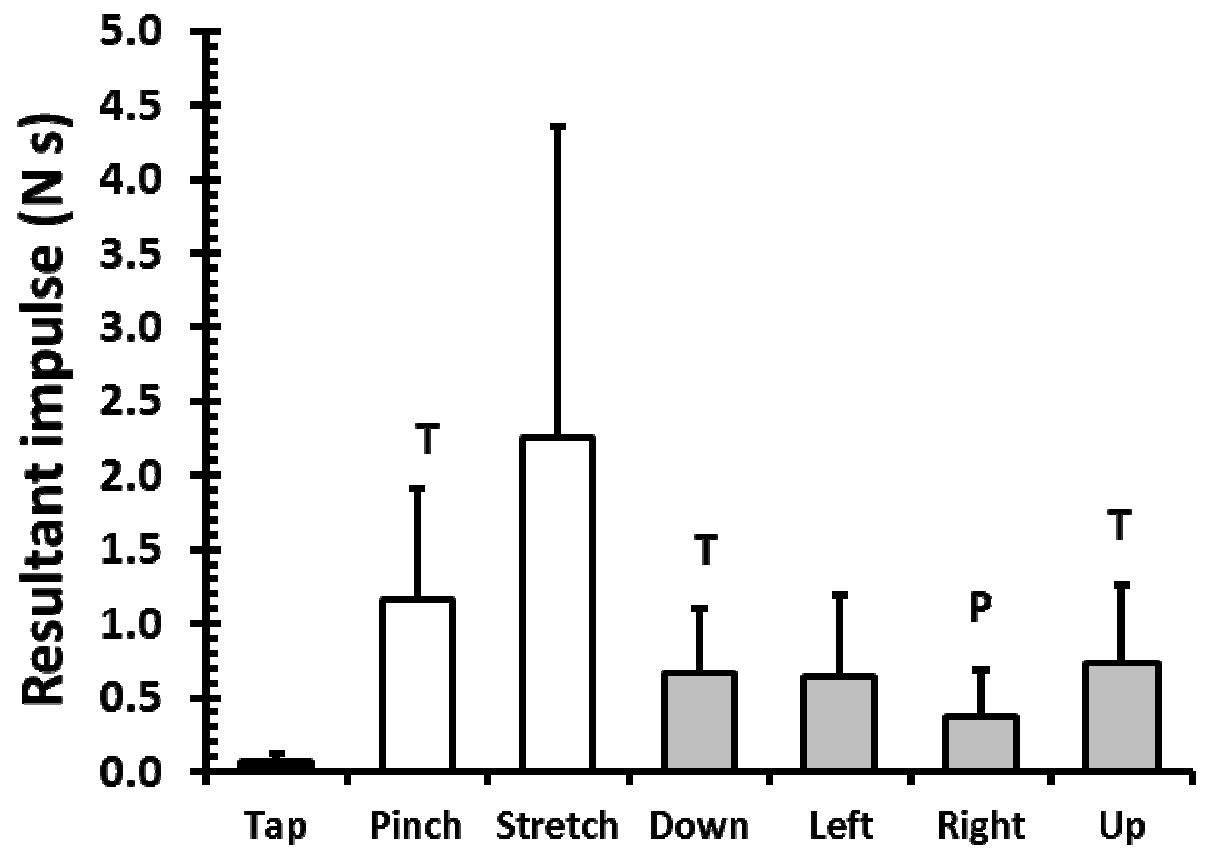


Fig. 8.

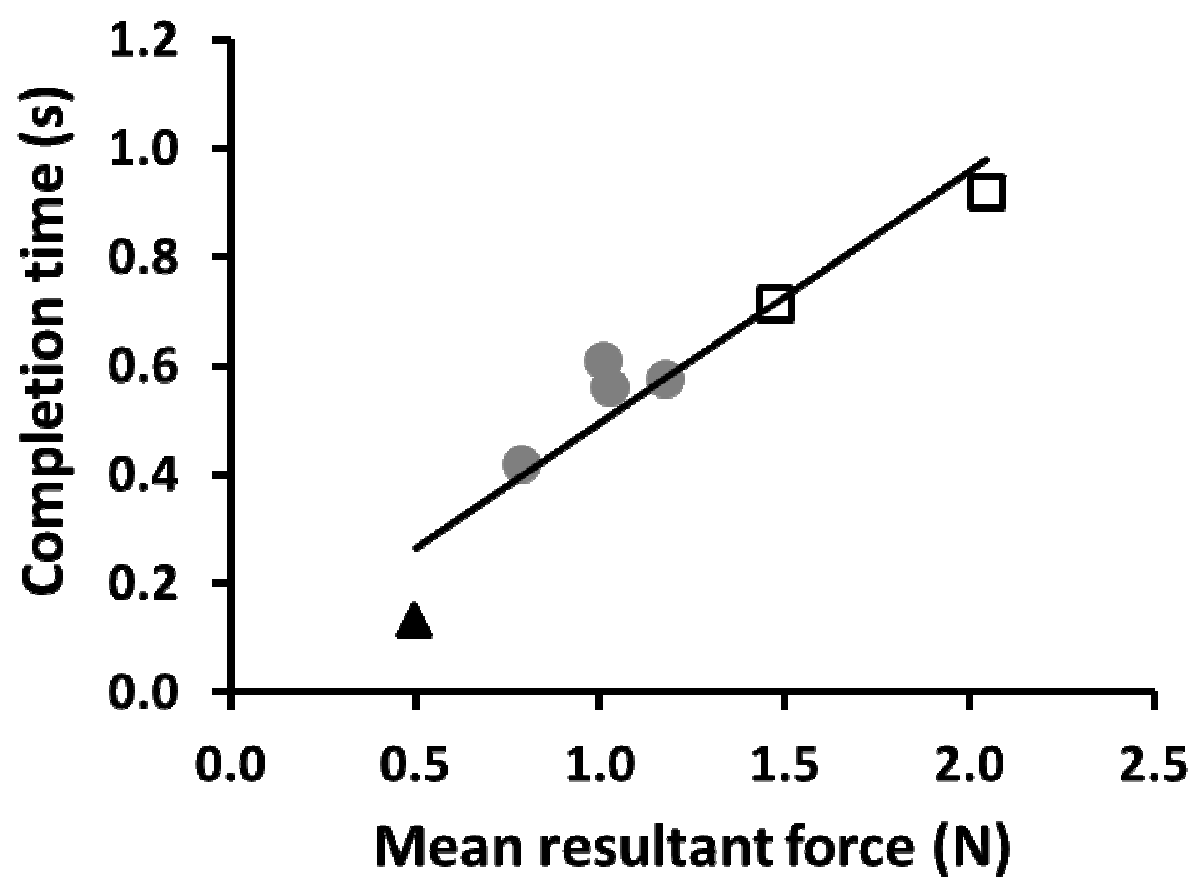


Table 1. Comparison of mean (standard deviation), coefficient of variation (CoV), and maximum values for forces, completion times and resultant force impulse measurements among touchscreen gestures.

\begin{tabular}{|c|c|c|c|c|c|c|c|}
\hline & Tap & Pinch & Stretch & Slide Down & Slide Left & Slide Right & Slide Up \\
\hline \multicolumn{8}{|c|}{ Resultant Force $(N)$} \\
\hline Average & $0.50(0.09)$ & $1.47(0.63)$ & $2.05(1.13)$ & $1.01(0.43)$ & $1.03(0.45)$ & $0.79(0.38)$ & $1.18(0.47)$ \\
\hline $\mathrm{CoV}(\%)$ & 18 & 42 & 55 & 43 & 44 & 49 & 40 \\
\hline Maximum & 3.33 & 3.78 & 6.7 & 2.45 & 3.44 & 2.35 & 3.34 \\
\hline \multicolumn{8}{|c|}{ Shear Force (N) } \\
\hline Average & $0.13(0.06)$ & $.277(0.20)$ & $0.37(0.21)$ & $0.79(0.39)$ & $0.78(.41)$ & $0.62(0.33)$ & $0.90(0.38)$ \\
\hline $\mathrm{CoV}(\%)$ & 49 & 71 & 58 & 49 & 53 & 54 & 42 \\
\hline Maximum & 0.283 & 0.62 & 0.731 & 1.40 & 1.50 & 1.25 & 1.90 \\
\hline \multicolumn{8}{|c|}{ Completion time (s) } \\
\hline Average & $\begin{array}{c}0.133(0.0 \\
83)\end{array}$ & $0.715(0.255)$ & $0.920(0.398)$ & $0.610(0.236)$ & $0.560(0.300)$ & $0.421(0.181)$ & $0.577(0.256)$ \\
\hline $\mathrm{CoV}(\%)$ & 63 & 36 & 43 & 39 & 53 & 43 & 44 \\
\hline Maximum & 0.319 & 1.227 & 1.651 & 1.059 & 1.190 & 0.840 & 1.059 \\
\hline Minimum & 0.032 & 0.416 & 0.391 & 0.323 & 0.264 & 0.227 & 0.234 \\
\hline \multicolumn{8}{|c|}{$\begin{array}{l}\text { Resultant Impulse } \\
(\mathrm{Ns})\end{array}$} \\
\hline Average & $0.07(0.06)$ & $1.16(0.75)$ & $2.25(2.11)$ & $0.66(0.44)$ & $0.64(0.55)$ & $0.37(0.32)$ & $0.73(0.53)$ \\
\hline $\mathrm{CoV}(\%)$ & 77 & 65 & 94 & 67 & 86 & 86 & 73 \\
\hline Maximum & 0.178 & 2.62 & 7.75 & 1.55 & 1.63 & 1.08 & 1.90 \\
\hline
\end{tabular}




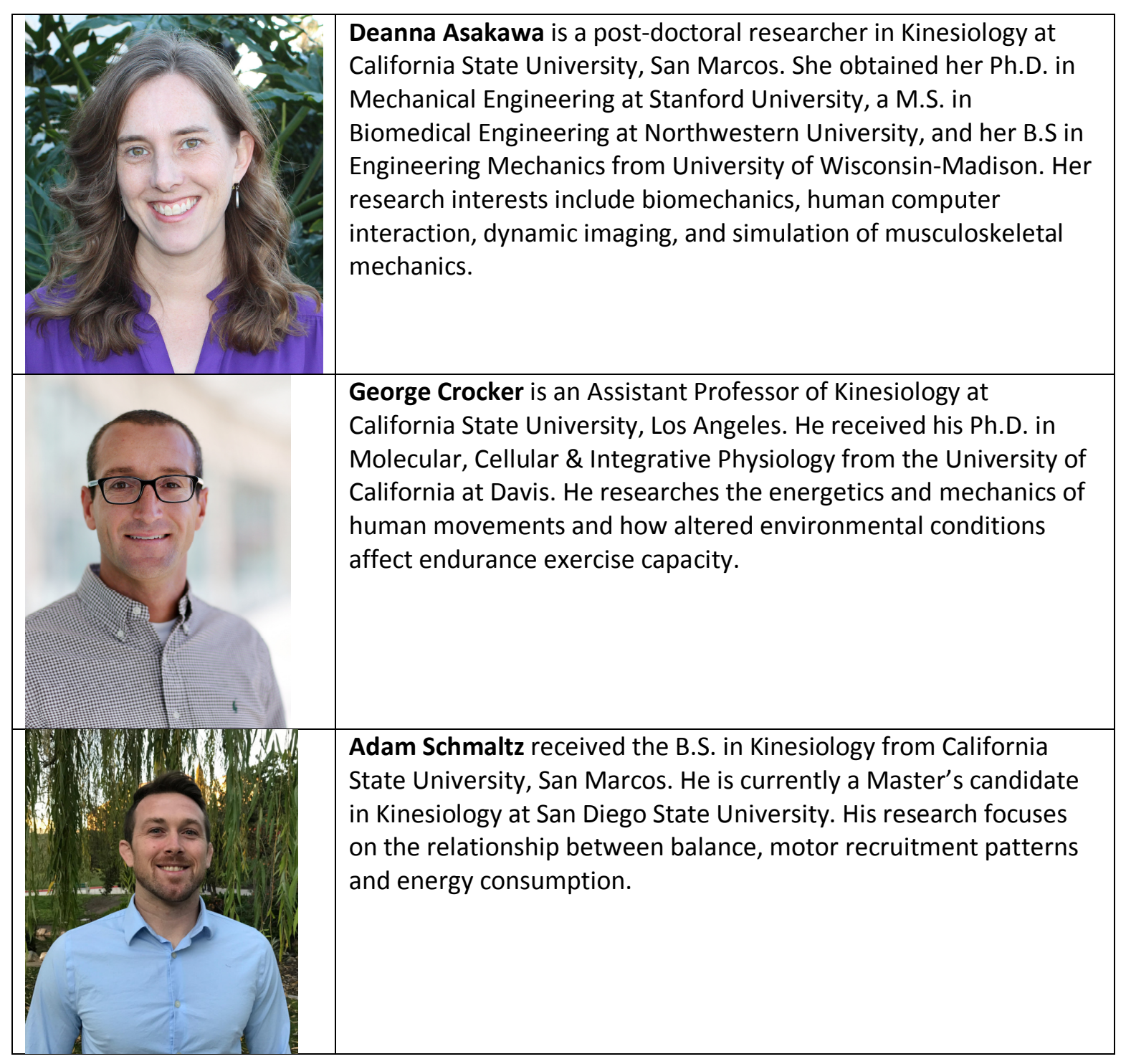




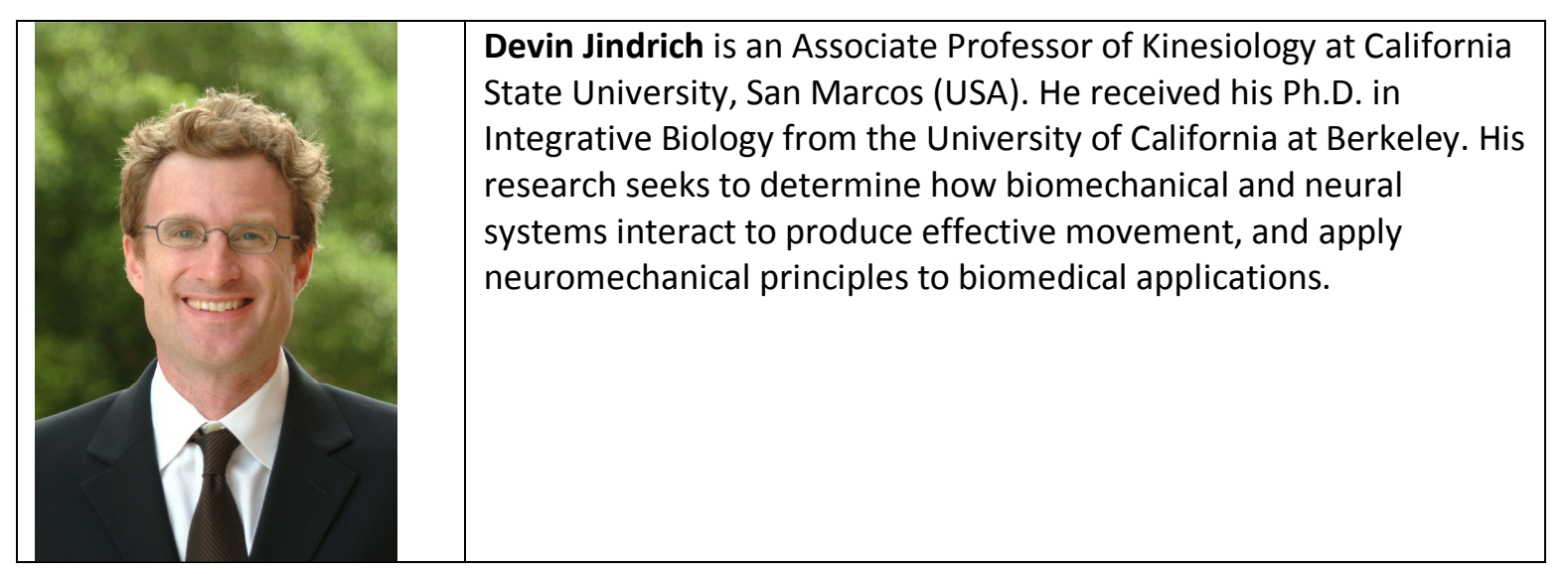

